\title{
The Application of Neural Network to Missile Fault Diagnosing System
}

\author{
Hu Jian \\ Aviation Maintenance School for NCO, Air Force Engineering University, China \\ 13523896492@163.com
}

Keywords: application; missile; fault diagnosing system; neural network

\begin{abstract}
Based on analyzing the incompleteness and uncertainty of information existing in missile fault diagnosing system, a new fault diagnosis method based on wavelet transform and neural network is proposed. The wavelet transform is used to pre-process data and extract fault feature vectors. The neural network is used to identify fault types. Diagnostic results of instance proved the effectiveness and superiority of the proposed method.
\end{abstract}

\section{Introduction}

The missile system is a kind of precision mechanical electrical system, with the rapid development of scientific technology, the scale and structure of missile system continue to become complicated. In order to achieve more excellent combating capability, the multiple guiding technology, the impetus vector and the pneumatic power multiple control technology are adapted in the missile system. In the process of missile system operation, natural and man-made interference often occurs and the failure is difficult to avoid. So the higher correlative theory level and abundant maintenance practice experience are required in the traditional missile fault diagnosing system. Therefore, the maintenance level will become lower and the maintenance experience will be wasted [1]. It is necessary that adopting effective method to diagnose the fault of missile system accurately, finding out the fault components, enhancing missile system combat efficiency and reducing economic losses, appear particularly important. Now, with the further development of artificial intelligence, especially machine learning, data mining, etc, many theories and methods are offered to diagnose the fault. Such as expert system, optimization method, fuzzy sets theory etc. Although these theoretical researches have scored some achievements, there are still certain limitations, for example, Fourier transform has been playing an important role in data processing, but Fourier transform has some defects, on the one hand, it can only analyze stationary signals, it cannot characterize sharp-variation signals that occur during faults diagnosing. On the other hand, Fourier transform cannot localize the singularities that always symbolize some sudden faults, and its frequency and time resolutions contradict each other.

As a powerful tool of signal analysis, wavelet transform has good localization properties in time and frequency domain, focus to any details of the analysis object with taking fine time or frequency step length of high frequency, express any changes existing in the object, so as to get accurate feature separation results from the measurement data with bad SNR [2, 3].

By using wavelet transform to separate the feature, the key process lies in the determination of optimal decomposition levels. On the one hand, we want to separate the feature components as far as possible, on the other hand, keep the fixed errors and true value apart from the separated feature. The current methods need either manual setting threshold control or results testing with extracted trend by wavelet transform, which increase the difficulty of the application of separation methods and raise the risk of error introduced. In accordance with the above case, the paper proposes a new method which approximates the feature with detail components of the wavelet decomposition, determines the optimal decomposition level on the frequency intervals between the feature and other components, then gets the feature directly. The method avoids the indirect error with modeling and indirect methods [4].

The uncertainty of missile system operation, the diversity, complexity and associated level-oriented of gathering information, cause detection randomness and uncertainty. Wavelet is a new developing signal processing means. It is localized both in time and frequency domains. So it is possible to characterize the local singularities based on the coefficients basis expansion. Combining 
model theory and statistical knowledge, wavelet provides a method to describe causal relationship between variables. Using probability theory to handle the uncertainty between different knowledge for conditions related, so it thus becomes one of the models in the field of uncertain knowledge representation and reasoning. Applying the neural network to missile fault diagnosing system, can solve incompleteness and uncertainty. Using the neural network structure learning algorithm to obtain a precise missile fault diagnosis model in qualitative, using the wavelet parameters learning algorithm to obtain the table of conditional probability and reflect the link degree between components in quantitative. Through reasoning algorithm further achieve the missile fault diagnosis under the uncertainty and incomplete information [5].

\section{Wavelet Transform}

The existing methods of random errors separation which using wavelet transforms mainly extract trends of the measurement data and get the random errors by subtracting the trend items, which is difficult to apply because it requires the precise modeling of the true values and fixed error. The paper implements the separation from another perspective, which directly uses the details of wavelet transform approximate the random error of measurement data, determines the wavelet decomposition levels based on the frequency bands character of the measurement data and separates the random errors directly which avoiding additional errors from the modeling process. The new method of this paper need not know the error models that the random errors or the fixed errors satisfy with. The only thing we should pay attention to is setting up the relationship between the frequency bands character of measurement data and the decomposition levels of the wavelet transform, and finding out the optimal decomposition level when the random errors has been separated entirely.

If a function $\psi(t)$ satisfies the following admissibility condition, it is called mother wavelet.

$$
\int_{0}^{+\infty} \frac{\psi^{2}(\omega)}{\omega} \mathrm{d} \omega=\int_{-\infty}^{0} \frac{\Psi^{2}(\omega)}{\omega} \mathrm{d} \omega<+\infty
$$

The families of functions are generated from $\psi(t)$ by the operation of dilation and translations form a basis or a frame of $a$ integrable square space $L^{2}(R)$. Such families are called wavelets. Wavelet transform is to expand a signal to this wavelet.

$$
\begin{aligned}
& \psi_{\mathrm{a}, \mathrm{b}}=|\mathrm{a}| \\
& W(a, b)=\int_{-\infty}^{+\infty} \psi\left(\frac{\mathrm{t}-\mathrm{b}}{\mathrm{a}}\right) \\
& \psi_{a, b}(t) f(t) d t
\end{aligned}
$$

$W(a, b)$ is the wavelet transform of $f(t)$. Here $a$ is called scale parameter and $b$ translation parameter. In order to calculate wavelet transform fast on computer, we must discretizate parameters $a$ and $b$. In digital signal processing, the dyadic discrete wavelet transform is most widely used. Make $a=2^{-j}, b=2^{-j} k$, where $j, k \in \mathrm{Z}$. From the theory of the multi-resolution analysis developed by Mallat, supposed that $\left\{V_{j}\right\}(j \in \mathrm{Z})$ is a multiresolution analysis of $L^{2}(R)$. There must be an unique scale function $\varphi(t)$, by dilation and translation of $\varphi(t)$, we can get a family of functions forming an orthonormal basis of $V_{j}$. The mother wavelet $\psi(t)$ can be deduced from $\varphi(t)$. And the family of functions forms an orthonormal basis of another set of closed sub-space $W_{j}$. $W_{j}$ is the orthogonal complement of $V_{j}$ in $V_{j+1}$, that is

$$
W_{j} \perp V_{j}, W_{j} \oplus V_{j}=V_{j+1}
$$

Mallat proved that the resolution of any actual signals can be normalized to 1 , that is, $f(t) \in V_{0}$. So $f(t)$ can be projected to the set of the sub-space to yield its orthogonal wavelet representation $A_{j}^{d} f(n)$, $D_{j}^{d} f(n)$, where $A_{j}^{d} f(n)$ is the projection of $f(t)$ on $V_{j}$, called approximation of $f(t)$. It can be interpreted as a low-pass filtering of $f(t), D_{j}^{d} f(n)$ is the projection of $f(t)$ on $W_{j}$, called detail signal. It can be interpreted as the filtering of $f(t)$ by a bank of band-pass filters. So wavelet transform can be viewed as extracting information on different frequency bands from the original signal and shows it on time axis. Thus we can view the signal characteristics on both time and frequency domains simultaneously. The high frequency information is shown on fine scales and the low frequency information on large scales. We can select transforms on proper scales to characterize the original 
signal according to our requirement. On this account wavelet transform is a powerful signal processing tool.

On the basis of the multi-resolution, Mallat developed a pyramid algorithm for discrete dyadic wavelet transform.

$$
\begin{aligned}
& A_{j}^{d} f(n)=\sum_{k=-\infty}^{\infty} h(2 n-k) A_{j}^{d} f(k) \\
& D_{j}^{d} f(n)=\sum_{\mathrm{k}=-\infty}^{\infty} g(2 n-k) A_{j}^{d} f(k)
\end{aligned}
$$

It has been proved that we can reconstruct the original signal completely from its wavelet representation $A_{j}^{d} f(n), D_{j}^{d} f(n)$. This indicates that wavelet transform contains almost all of the original signal information. So it is viable to extract features by wavelet transform. From DWT if there are $N$ data in the original signal, the lengths of $A_{j}^{d} f(n)$ and $D_{j}^{d} f(n)$ are $2^{j} N(j<0)$ respectively. That is, with increase of the scale, their lengths decrease in proportion to 2 . Therefore, wavelet transform can compress data efficiently.

In fault diagnosis, the length of the feature vectors should be as small as possible under the condition of characterizing the faults accurately. Considering singularities of the fault signals, we take advantage of wavelet transform that is localized on both time and frequency domains, to extract a certain period as our diagnosing feature vector. To compare with each other, we study the following two feature extracting measures, the first is to extract $A_{-1}^{d} f$ as a feature vector. The difference between original signal and $A_{-1}^{d} f$ is $D_{-1}^{d} f$. $D_{-1}^{d} f$ mostly contains the noise information in high frequency domain. When it is discarded, SNR will increase greatly, and the data number will decrease half. The second is to extract $A_{-2}^{d} f$ from $A_{-1}^{d} f$. Together with the mean vaule, mean square vaule and variance of $A_{-2}^{d} f$ which characterize the lower frequency information of $A_{-1}^{d} f, A_{-2}^{d} f$ constructs another type of feature vector. Using this vector we will lose some information in low frequency domain, but its demension is only about a quarter of that of the original signal[6,7].

When we regard these two feature vectors as the learning and testing sets of neural network system, the number of the neurons will decrease greatly comparing with choosing the original signal as the input samples. It has been turned out by some experiments that these measures are efficient and accurate in missile fault diagnosing system.

\section{Missile Neural Network Fault Diagnosing System}

The missile system is composed of the guiding system, fuse-battle system, thruster, power system and missile body system. The guiding system is used to process all kind of information from the target, the missile and the environment. The fuse-battle system is divided into the fuse and battle part, it is used to attack the target directly.The missile thruster is used to provide the flighting power so as to attain the speed and voyage for missile. The power system is composed of the electric power subassembly in missile, the gas power subassembly, the hydraulic pressure subassembly and so on.It is used to provde power for the other parts. Such above parts are assembled by the missile body system, it must have good pneumatic shape.

The missile neural network fault diagnosing system bases on the pre-processing method by means of the learning function, the large-scale parallel distributed memorizing and processing capabilities, as well as the collective operating capability of the neural network, accomplishes the fault diagnosis of missile systems. At last, accomplishes the adaptive inference and improve the real-time processing ability and adaptability of the missile systems.

In the missile neural network fault diagnosing system, at first, we should construct the neural network model, secondly, decide the learning algorithm according to the characteristic of the missile system, finally, study the problems relating to the fault diagnosis.

Selection of the diagnosing parameter. The characteristic parameters will be much more changed when the missile system fault occurs. Therefore, it is not in light of abnormal diagnosis on absolute 
value as far as possile. It is regarded as the initial to normal value ratio as testing parameter on the basis of fault diagnosis. The monitoring parameter is defined as following.

$$
\mathrm{F}\left(\mathrm{x}_{\mathrm{i}}\right)=\mathrm{S}_{\mathrm{T}}\left(\mathrm{x}_{\mathrm{i}}\right) / \mathrm{S}_{\mathrm{R}}\left(\mathrm{x}_{\mathrm{i}}\right)
$$

Where, $S_{T}\left(x_{i}\right)$----the relative testing state parameter value of $x_{i}, S_{R}\left(x_{i}\right)$---- the relative normal state parameter value of $x_{i}$.

$\mathrm{S}_{\mathrm{T}}\left(\mathrm{x}_{\mathrm{i}}\right)$ to $\mathrm{S}_{\mathrm{R}}\left(\mathrm{x}_{\mathrm{i}}\right)$ ratio is compared with the threshold $\mathrm{A}$, if $\mathrm{S}_{\mathrm{T}}\left(\mathrm{x}_{\mathrm{i}}\right) / \mathrm{S}_{\mathrm{R}}\left(\mathrm{x}_{\mathrm{i}}\right)>\mathrm{A}$, then missile fault sign $\mathrm{w}=1$, that is to say, the missile fault sign exists, otherwise, the missile fault sign does not exist.

Establishment of the knowledge system warehouse. The neural network expert knowledge system can be set up with producing rule, that is to say, every weight and threshold in the neural network are defined. It is decomposed into the knowledge acquisition and storage. The knowledge acquisition process is shown with training sample gaining and selection. The training sample stems from the characteristic parameter when the same type diagnosing missile systems operate normal and abnormal. The knowledge storage process is that store the successive weights and thresholds trained the neural network with the training sample. Therefore, the knowledge system warehouse is formed.

Firstly, the fault knowledge structure is analysed in accordance with the diagnosing object characteristic. The neural network structural model is resolved according to fault portent and sample number, that is to say, the input, output and implicative neural cells number are decided.

Secondly, the neural network training sample is selected in accordance with the diagnosing object characteristic and missile expert experience.

Thirdly, the knowledge system warehouse is formed by training with training samples, the neural network selecting weight and threshold are gained. The knowledge system warehouse is used to store some connecting weights expressed with numberal and implicative form rather than direct rule.

Selection of the neural network structural diagram. At present, there are many neural network models, we can select the self-organizing neural network and the multi-level feed forward neural network model. The ART neural network model can be adopted to perform the multiresolution. Now, we adopt the ART neural network model in diagnosing the fault of missile system in order to enhance the resoning and learning ability[8]. The schematic diagram of the missile neural network fault diagnosing system is shown in Fig.1.

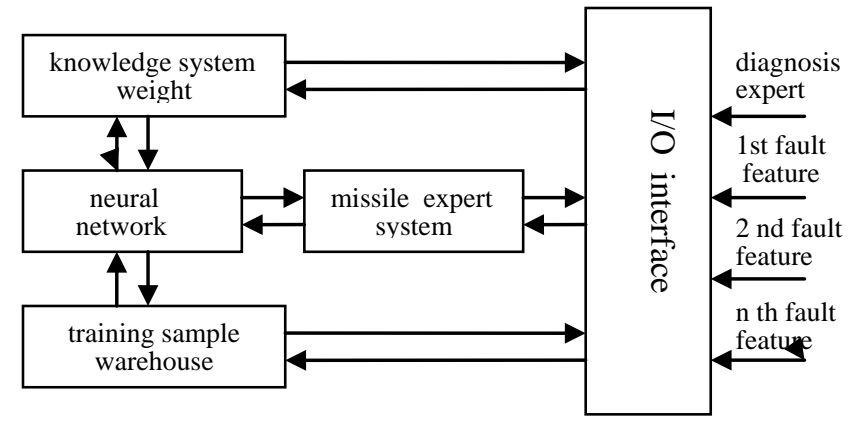

Fig.1. The schematic diagram of the missile neural network fault diagnosing system

ART neural network model consists of the input and output nerve cells, it computes the matching score, that is, the output values of the output nerve cells, through the feed forward connecting weights and sample input. The nerve cell activity with the largest matching score is enhanced through the lateral inhibitting between the output nerve cells. The other output nerve cells activity degrees are gradually decreased. Nerve cells represent radom variables, the edges among nerve cells represent the relationship between cells. The conditional probability express relationships strength. The variable can be the abstract of any problem, such as test value, observations, etc. The feedback connecting from the output nerve cell to the input nerve cell performs learning and comparing. ART neural network model can be discribed with the differential equation and perform self-organization with the competitive learning[9].The adjacent processing units restrain one another, finally, only one processing unit is active, this active processing unit represents the present type of the fault in power system. Therefore, it is applicable to express and analysis of uncertainty and probabilistic events, applied to a conditional relies on multiple controlled factor of decision making, can make reason among incomplete, inaccurate or uncertain knowledge or information. 
The architecture of the ART neural network model can be divided into the caring subsystem and adjusting subsystem[10]. By means of the two mutual compensating subsystems and interaction of the controlling mechanism, the fault data is obtained. The caring subsystem solves the evaluation of the missile system performance in the known environment, for example, the line, transformer and bus. The adjusting subsystem solves the uncertainty and incompleteness in the missile system. The following kind of system is considered. Because the ART neural network model is the kind of self-organizing neural network model, it forms the response to input through competition. In the application of the neural network to the fault diagnosis of the missile systems, we need the pre-processing network that the features characterizing original signals are extracted.

Learning the neural network, we can obtain the qualitative information and quantitative information. The qualitative information is reflected by arc, representing the relationships among variables; the quantitative information is reflected by relationship strength among variables, namely represented by the table of conditional probability. Uncertainty reasoning methods mostly developed on the basis of uncertainty reasoning method, or rather to expand or improve certainty reasoning. The most common way is to put instructions degree of certainty data attached to reasoning rules. Neural network reasoning actually know results obtained parameters process, fault diagnosis reasoning is to obtain the reason of fault by using the fault information, says variables and the state of the fault occured in event joint probability is the largest. The fault diagnosis model established with neural network can clearly express the relationship among components, all levels of the protection and the breakers.

In the missile fault diagnosing system based on the neural network, the neural network inputs are some information collected missile fault character, and train the neural network by use of the representative training sample extracted from the engineering practical example, and infer the missile fault by use of neural network. When the learning sample is not accepted by neural network, the missile experts infer the missile fault, and then input the learning sample into the training sample warehouse, adjust the weight and continue learning the network until obtain the correct inference.

\section{Simulation and Conclusion}

In order to demonstrate the fault diagnosis method established with neural network in the missile system, the following conditional parameters are selected. The missile electric system fault diagnosis includes: line, appliance and system. When the missile system is tested, the main tested capability parameters are as follows: Sound Signals, they are used to judge the tested capability of missile. Peg-top Frequency, it is used to assure the peg-top startup and work normally conditions, then the missile tracking target capability can be judged. Track Angular Velocity and Track Range, they are used to judge the tracking capability and abaxial capability of the guiding system. Moment parameters, by it the rudder working conditions can be judged, and the moment parameters can be divided to remain zero moment, zero moment and slop moment. Delay, by it the safe devices and uninsured devices working conditions can be judged. Power Parameters, they include the voltage, current and the power frequency. Fuse-battle System Parameters, they are composed of touch fuse circuit, short range fuse circuit and self-destruction fuse circuit. Igniting Circuit Resistances, they are composed of engine igniting circuit resistances, power igniting circuit resistances, and uninsured devices igniting circuit resistances [11].

Relay protection of lines and appliances protection is 3-sectional protection: main protection only protect circuit itself, the scope of protection is generally $75 \%$ of the full-length. The first backup protection protects the whole length of circuits, when the main protection doesn't start, the first backup protection start automatically. The second backup protection, the adjacent elements fault but the protection doesn't start, the second backup protection starts automatically to remove fault. System relay protection is only main protection, when the system is fault, relay protection automatically start [12].

In this paper, three types of faults are analyzed. We do 3-scale wavelet transform on 256 data for every fault sample and extract feature vectors as discussed above. At first, we regard $A_{-1}^{d} f$ of every 
sample as a learning set and input it to neural network, then we regard $A_{-2}^{d} f$ and the mean value, mean square value and variance of $A_{-2}^{d} f$ of every sample as a learning set and input it to neural network. Among the faults diagnosis, the maximum fault probability is obtained, therefore, the diagnosing result is obtained and given in Tab.1.

Tab.1 Diagnosing Result

\begin{tabular}{|c|c|c|c|c|}
\hline No. & fault1 & fault2 & fault3 & diagnosis \\
\hline 1 & 0.98453 & 0.00570 & 0.01769 & fault1 \\
\hline 2 & 0.00534 & 0.99656 & 0.00728 & fault2 \\
\hline 3 & 0.00321 & 0.00512 & 0.96608 & fault3 \\
\hline
\end{tabular}

From the result, we can see that by both of the two feature extraction methods, the three types of faults are identified correctly. The fault features extracted from wavelet transform can characterize original signals accurately with the data quantity greatly compressed. At the same time, the noise is restrained. If these fault feature vectors are used as neural network input sets, the construction of the neural network will be terrible simplified and the calculation time obviously shortened. This makes it possible to use this method to on-line fault diagnosis. It is proved by experiments that this measure is especially efficient in diagnosing some faults.

\section{References}

[1] Mingqing Xiao. Airborne Missile Test Theory. Beijing:National Defence Industry Press, 2011.

[2] Qingxian Jiang. Robust Estimation for FOG Data Based on Wavelet Threshould De-Noising. Chinese Journal of Sensors and Actuators, vol.22, pp. 1595-1601, November, 2009.

[3] Lihua Guo. Application of Wavelet Transform in Random Error Separation of Telemetered Data. Journal of Projectiles, Rockets, Missiles and Guidance, vol. 31, pp. 249-252, August, 2011.

[4] Lifeng Zhou. A Study on Error Analysis of Radar Measurement Data in Proving ranges. Radar \& Ecm, pp.4-7, 2008.

[5] Xinlong Wang. Investigation of Random Error Model for Fiber Optic Gyroscope. Journal of Beijing University of Aeronautics, vol.32, pp. 769-772, July, 2006.

[6] Guofeng Yang. Research on Power System Fault Diagnosis Based on Bayesian Network. Proceedings of the ICCSEE2013, pp. 2553-2556, March, 2013.

[7] Fushun Wen. Fault Sections Estimation in Power System Using Genetic Algorithm and Simulated Annealing. Proceedings of the CSEE, pp. 29-35, May, 1994.

[8] Dong Chen. Applications and Research of the Bayesian Network in Power System Fault Diagnosis. Shenyang University of Technology Press, 2009.

[9] Feng Ding. Neural Network Expert Systems [M]. Beijing: Science Press, 2006.

[10] Shie-Jue Lee. An ART-Based Construction of RBF Neural Networks. IEEE Transactions on Neural Networks, vol.13,no.6,pp.1308-1321, November, 2002.

[11] Xiaoyu Zhang. Research on Faults Diagnosis System of an Air-to-ground Missile Based on Wavelet Neural Network. Proceedings of the AMCCE2015, pp. 2300-2305, April, 2015.

[12]Weibing Li. Research on Comprehensive Fault Diagnosis Technology of Tactical Missile. Tactical Missile Technology, vol.4, pp. 42-46, August, 2011. 